\title{
Study of the modulation of Galactic positrons and electrons in 2006-2016 with the PAMELA Experiment
}

\section{V.V. Mikhailov, ${ }^{a, *}$ S.Y. Aleksandrin, ${ }^{a}$ S.A. Koldobskiy, ${ }^{a}$ M. Boezio, ${ }^{b}$ R. Munini,,${ }^{b}$ O.P.M Aslam, ${ }^{c}$ D. Bisschoff, ${ }^{c}$ M.D. Ngobeni ${ }^{c, d}$ and M.S. Potgieter ${ }^{e}$ on behalf of the PAMELA Collaboration}

(a complete list of authors can be found at the end of the proceedings)

${ }^{a}$ National Research Nuclear University MEPhI, 31 Kashirskoe shosse, Moscow. Russia

${ }^{b}$ INFN, Sezione di Trieste, Trieste, Italy

${ }^{c}$ Centre for Space Research, North-West University, Potchefstroom, South Africa

${ }^{d}$ School of Physical and Chemical Sciences, North-West University, Mmabatho, South Africa

${ }^{e}$ Institute for Experimental and Applied Physics, CA University in Kiel, Germany

E-mail: vvmikhajlov@mephi.ru

The PAMELA experiment had operated almost ten years on board of the Resurs DK1 satellite. The satellite was launched on the $15^{t h}$ of June 2006 and placed in orbit with an inclination of $70^{\circ}$ and an altitude of 350 to $610 \mathrm{~km}$. The experiment had continuously measured electron and positron fluxes of Galactic cosmic rays in a wide energy range from about $50 \mathrm{MeV}$ to hundreds of $\mathrm{GeV}$. The spectra of electrons have been analysed from the end of $23^{r d}$ until the beginning of $24^{\text {th }}$ solar cycle including the prolonged deep solar minimum period from 2006 to the end of 2009 and the solar magnetic polarity reversal period in 2012-2014. Here, we present updated annual electron spectra from 2006 to 2015.

$37^{\text {th }}$ International Cosmic Ray Conference (ICRC 2021)

July 12th-23rd, 2021

Online-Berlin, Germany

\footnotetext{
*Presenter
} 


\section{Introduction}

New experimental efforts have been undertaken to precisely measure cosmic ray electron and positron energy spectrum. Measurements of electron spectra at high energies have been performed by orbital experiments such as Fermi-LAT [3] and DAMPE [4] AMS-02 [2] and CALET [5]. These spectra give important information about particle propagation in the Galaxy. At lower energies spectra of electrons and positrons are modulated in the heliosphere and their measurements are important to study and understand the propagation of these particles in the interplanetary magnetic field (IMF). With the exception of some balloon flights e.g. [10], long duration, simultaneous and precision measurements, separating electron and positron fluxes effectively below $1 \mathrm{GeV}$, have not been carried out so that published measurements have been controversial. However, the PAMELA mission [6] has filled this shortcoming in the investigation of cosmic ray electrons and positrons, and how differently they are being modulated in the heliosphere over a solar cycle.

The magnetic spectrometer PAMELA was launched on board the Russian Resurs-DK1 satellite on the $15^{\text {th }}$ of June 2006 and it had been continuously gathering data for almost 3200 days, until $24^{\text {th }}$ of January 2016; its total lifetime was thus quite long, almost 10 years. The main goal of the experiment was to study the energy spectra of cosmic ray antiparticles over a wide energy range so the apparatus was equipped with a set of detectors that allowed precision particle identification. The satellite had an initial quasi-polar $\left(70^{\circ}\right.$ inclination) elliptical orbit at altitudes between 350 and $600 \mathrm{~km}$ [6]. Particle energy may be inferred from rigidity in the magnetic spectrometer or from energy deposit in the calorimeter together with independent measurement of particles' velocity with a Time-of-Flight system. Results of the PAMELA observation of electron and positron fluxes which had been made during the first years of the flight (2006-2009), were reported by [1, 11, 14] whereas the positron to electron ratio above $0.5 \mathrm{GV}$ was reported by [12]. Separate measurements of electrons and positrons need very strong requirements for track reconstruction to background contamination, mainly from protons, that leads to efficiency suppression. Moreover, the acceptance of the instrument decreases at low energies due to magnetic field of the spectrometer equipped with a permanent magnet that strongly deflects electrons and positrons below $0.05 \mathrm{GeV}$ out of the detection volume.

Here, we first report on a new analysis of electron data that is been performed to obtain their spectra below about $20 \mathrm{GeV}$ using PAMELA data collected from June 2006 to January 2016. The analysis will be continued to include positrons with the purpose to provide an update of the electron to positron ratio over a wide energy range over this period.

\section{PAMELA spectrometer}

The instrument consists of a Time-of-Flight system (ToF), an anticoincidence system, a magnetic spectrometer, an electromagnetic calorimeter, a shower tail scintillator and a neutron detector $[1,6]$. The ToF system provides the main trigger for particle acquisition, measures the absolute value of the particle charge and its flight time while crossing the apparatus (the accuracy is about $350 \mathrm{psec}$ ). Particle rigidity is determined by the magnetic spectrometer, composed by a permanent magnet with a magnetic field intensity $0.4 \mathrm{~T}$ and a set of six double micro-strip silicon planes to measure $\mathrm{X}$ and $\mathrm{Y}$ coordinates of particles tracks. The spatial resolution of the tracker system of the 
spectrometer was observed to be about $\sim 4 \mu \mathrm{m}$, corresponding to a maximum detectable rigidity (MDR) exceeding $1 \mathrm{TV}$. The tracker also provides measurements of ionization energy losses $d E / d x$ in six silicon planes. The high energy electron and positron identification is provided mainly by the electromagnetic imaging calorimeter. The calorimeter consists of 44 layers with strip silicon detectors interleaved by 22 tungsten planes. Total thickness is 16.3 radiation and 0.6 nuclear interaction lengths. Particles not cleanly entering the PAMELA acceptance are rejected by the anticoincidence system. The neutron detector and shower scintillator improve particle identification. The main axis of the PAMELA instrument was pointed mainly to the zenith during the flight, so any atmospheric effects could not affect observational results. The acceptance is about $21.6 \mathrm{~cm}^{2} \mathrm{sr}[1,6]$.

\section{Data analysis}

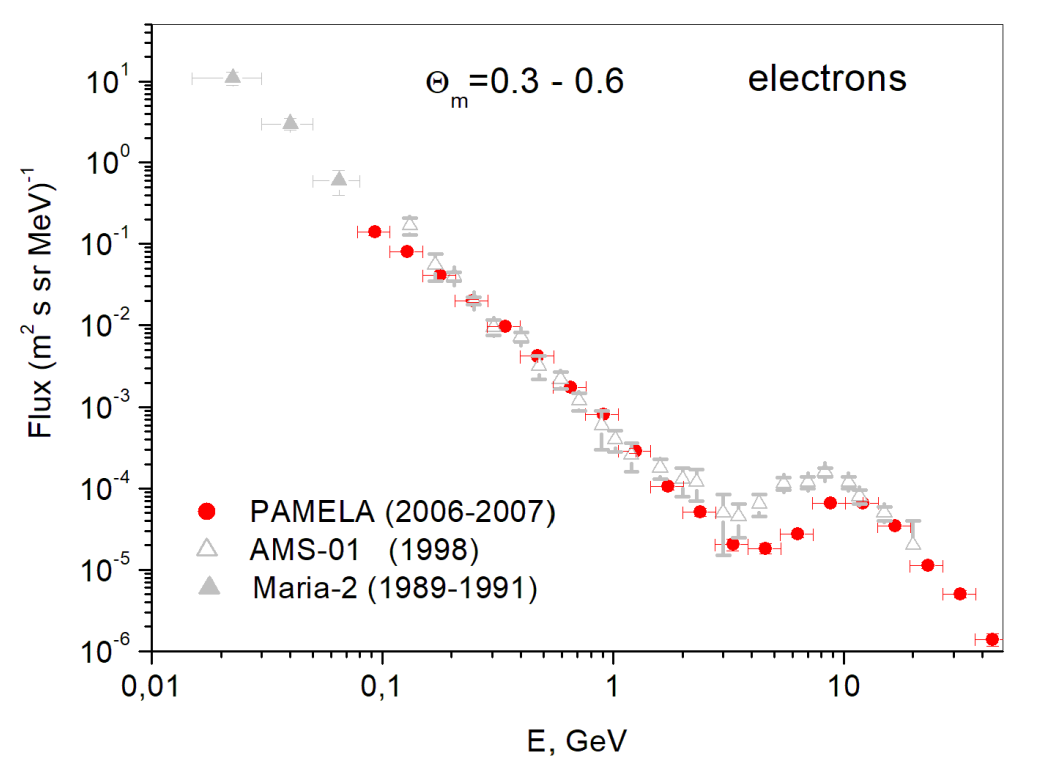

Figure 1: Differential energy spectra of electrons measured by PAMELA, MARIA-2 and AMS-01 [7] experiments at geomagnetic latitudes of 0.3-0.6.

The analysis was based on a full data set of the PAMELA experiment, using the number of tracks and energy losses in the magnetic spectrometer planes; rigidity, time of flight, set of calorimeter variables dealing with point of interaction and transversal and longitudinal profiles were all accessed for particle identification and energy determination. Electrons and positrons were selected requiring to have $d E / d x$ energy losses in the spectrometer planes and ToF detectors corresponding to charge $Z=1$ and particle velocity $\beta>0.8$. with no signals in the anticoincidence system, neutron detector and shower scintillator. To reduce background from wrongly reconstructed tracks, a consistency check of selected events was performed between tracker, calorimeter and ToF data. Proton rejection power and electron and positron efficiencies were estimated on the base of a Monte-Carlo simulation with PAMELA collaboration software [1]. To reduce the influence of the 
geomagnetic field, only events that were registered in the polar regions of the satellite's orbit were taken into account. For this analysis Rcut $<0.15 \mathrm{GV}$ was adopted.

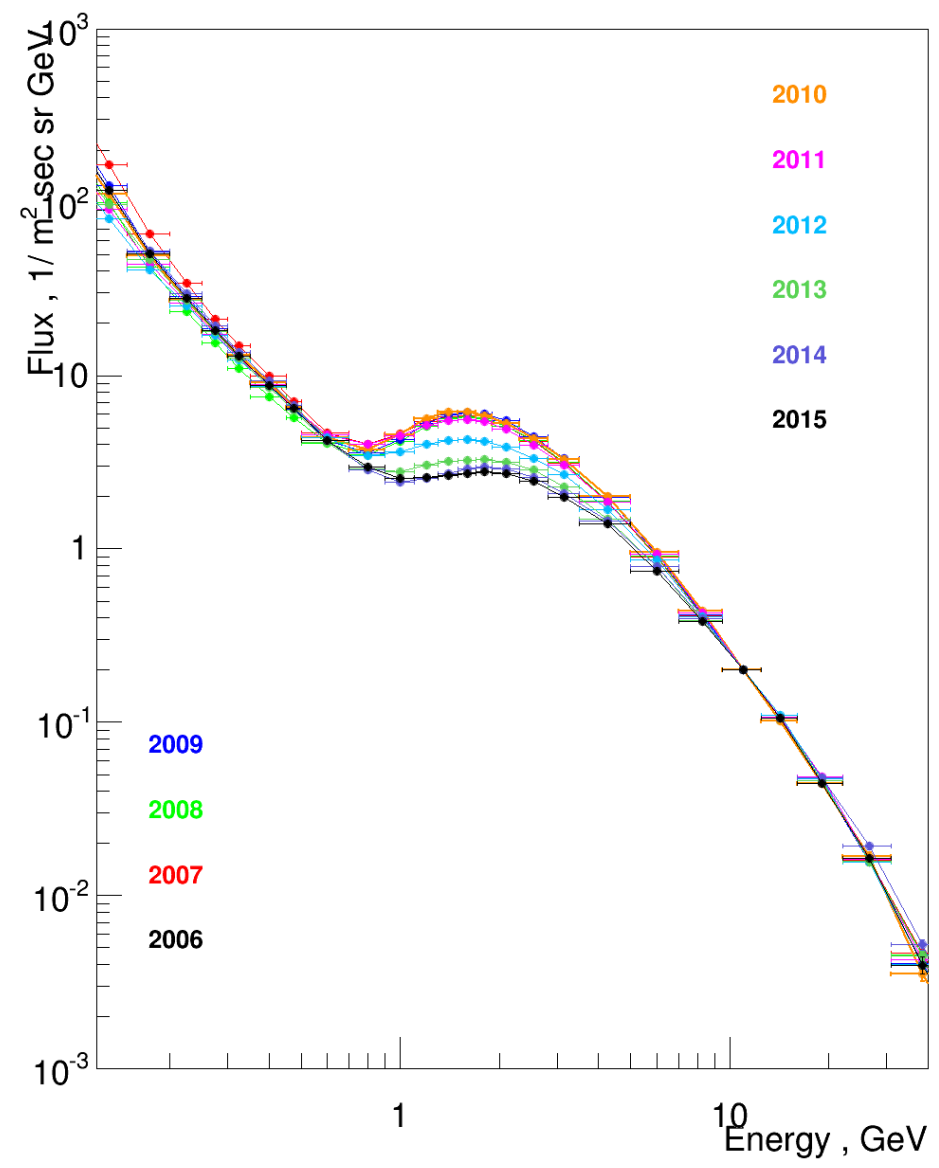

Figure 2: Differential energy spectra of electrons observed by PAMELA at middle latitudes $L=2-4$ (mean geomagnetic cutoff $R c u t$ about $1.5 \mathrm{GV}$ ) from 2006 to 2015 as indicated by varying colours.

For such a long duration flight the efficiency of the instrument may have varied with time. The flight efficiency of PAMELA was estimated from experimental data by using collective information from imaging calorimeter, magnetic spectrometer and ToF system. Quality of the efficiency estimation was verified with secondary undercut-off fluxes shown in Fig. 1. It is known that albedo particles originate from the inelastic interactions of cosmic ray nuclei in the residual atmosphere. Because of the relatively high threshold of pion productions and shielding by the Earth magnetic field, the flux of secondary particles is practically constant and do not vary significantly during the solar cycle [13]. This follows from Fig. 1 where measurements of different instruments form different epochs are presented.

Fig. 2 shows annual electron fluxes measured by PAMELA at middle latitude in L-shell intervals $=2-4$. There are variations of fluxes at rigidities around $1 \mathrm{GV}$ due to penumbra effects where cosmic ray particles below the geomagnetic cut-off can be observed. In this energy range 
solar modulation plays a particular important role varying significantly with time, although less so al lower energies while at much higher energies fluxes are changing progressively less with time as expected from solar modulation becoming less effective; see the descriptions of these modulation processes by $[15,16]$.

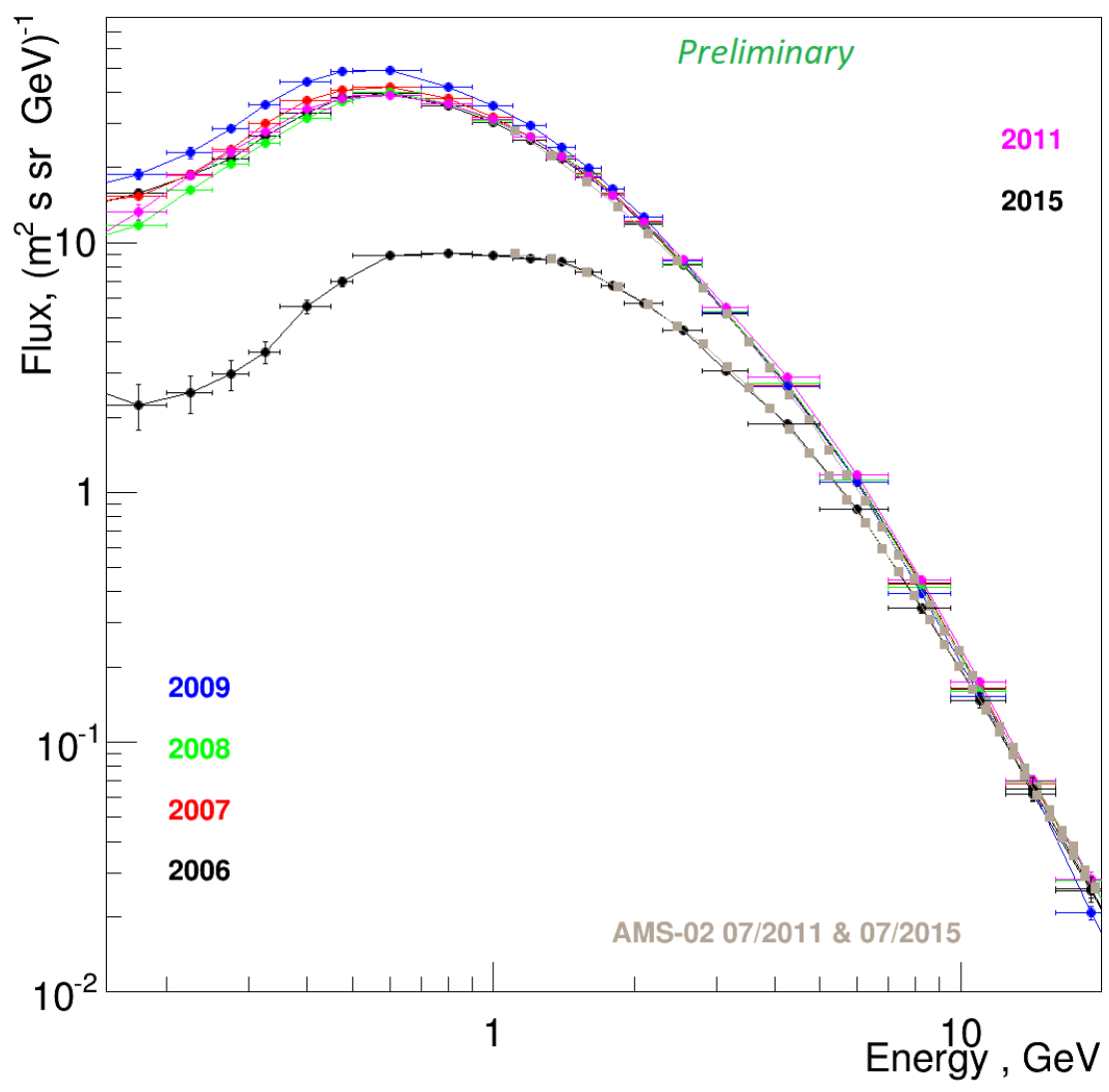

Figure 3: Annual differential energy spectra of electrons measured by PAMELA spectrometer in polar regions, L-shell > 10. AMS-02 data from [9]

\section{Results}

The annual differential spectra of Galactic electrons are shown in Fig. 3 at lower energies, for the range $0.15-20 \mathrm{GeV}$, averaged over the years from 2006 to 2009 and for 2011 and 2015. Despite the fact that the PAMELA magnetic spectrometer has a relatively small geometrical factor, spectral features are clearly visible in the figure. As noted, measurements of electron spectra for 2006 to 2009 were previously reported by [11] and the electron to positron flux ratios by [12]). As seen from Fig. 3, maximum electron flux was observed in 2009, which coincided with the time of the minimum of the $23^{r d}$ cycle of solar activity. At the same period a local peak in the positron and electron ratio was observed [12]. The electron flux began to decrease from the beginning of 
2010. It is interesting to mention that from 2011 to 2013, the ratio of positron and electron fluxes had remained practically constant, up to the reversal of the IMF polarity in 2013-2014, despite the increase in solar activity. In 2014-2016 there was a noticeable, almost 1.5 times, increase in the ratio of positron and electron fluxes at energies below about 2 to $3 \mathrm{GeV}$. However, from our preliminary estimations, electron fluxes in 2014 and 2015 are close, that is, the observed increase in the ratio during this period is associated with the first turn with an increase in the flux of positrons, which corresponds to theoretical predictions [17]. However, to obtain good agreement between theoretical calculations and experimental results, it was necessary to refine the local interstellar spectrum at the boundary of the heliosphere (heliopause), as well as the parameters of the model for a more accurate determination of the contribution the drift process.

\section{Acknowledgements}

We acknowledge partial financial support from Russian Foundation for Basic Research (RFBR Grant no: 19-52-6003) and the SA National Research Foundation (NRF Grant no: 118915) under the Joint Science and Technology Research Collaboration between SA and Russia. DB and OPMA acknowledge the financial support from the NWU post-doctoral programme. RM acknowledges partial financial support from the INFN Grant "giovani”, project ASMDM.

\section{References}

[1] O. Adriani, G.C. Barbarino, G.A. Bazilevskaya, and PAMELA collaboration, Phys. Rep. 544, 3232014.

[2] M. Aguilar, D. Aisa, B. Alpat, et al. Phys. Rev. Lett. 113, 2211022014.

[3] S. Abdollahi, M. Ackermann, M. Ajello, et al. Phys. Rev. D. 95, 0820072017.

[4] G. Ambrosi, Q. An, R. Asfandiyarov, et al. Nature 552, 632017.

[5] O. Adriani, Y. Akaike, K. Asano, et al. Phys. Rev. Lett. 120, 2611022018.

[6] P. Picozza, A. M. Galper, G. Castellini, and PAMELA collaboration, Astropart. Phys. 27, 296-315 2007.

[7] J. Alcaraz, et al. Phys. Lett. B, 484, 10, 2000.

[8] M, Aguilar M., L. Ali Cavasonza, G. Ambros et al. Phys.Rev. Lett. 121, 051102, 2018.

[9] M. Aguilar, L. Ali Cavasonza, B. Alpart, et al. Phys. Rev. Lett. 122, 1011012019.

[10] S. Mechbal, P.-S. Mangeard, J. Clem, et al. Astrophys.J. 903, 212020.

[11] O. Adriani , G.C. Barbarino , G.A. Bazilevskaya, and PAMELA collaboration, Astrophys. J. $810,2,142,2015$. 
[12] R. Munini, O. Adriani G.C. Barbarino, and PAMELA collaboration, Phys. Rev. Lett. 116, $241105,2016$.

[13] V. Mikhailov, O. Adriani, G.C. Barbarino et al. Bull. Russ. Acad. Sci. Phys. 81, 2, 2032017.

[14] V. Mikhailov, O. Adriani, G.C. Barbarino et al. Bull. Russ. Acad. Sci. Phys. 83, 8, 9742019.

[15] M.S. Potgieter and E.E. Vos, Astro. Astrophys. 101, A23, 2017.

[16] M.S. Potgieter, Adv. Space Res. 60, 848, 2017.

[17] O.P.M. Aslam, D. Bisschoff, M.D. Ngobeni, et al. Astrophys. J. 878, 1, 8, 2021. 


\section{Full Authors List: PAMELA collaboration}

O. Adriani ${ }^{1,2}$ G.Barbarino ${ }^{3,4}$ G.A.Bazilevskaya ${ }^{5}$ R Bellotti ${ }^{7,21}$ M. Boezio ${ }^{6}$ E.A.Bogomolov ${ }^{8}$ M.Bongi ${ }^{1,2}$ V.Bonvicini ${ }^{6}$ A.Bruno ${ }^{1,2}$ F.S.Cafagna ${ }^{7}$ D.Campana ${ }^{4}$ P.Carlson ${ }^{9}$ M.Casolino ${ }^{10,19}$ G.Castellini ${ }^{12}$ C.De Santis ${ }^{10}$ V.Di Felice ${ }^{10}$ S.V.Koldashov ${ }^{11, d}$ S.A.Koldobskiy ${ }^{11}$ A. N. Kvashnin ${ }^{5}$ A.Leonov ${ }^{11}$ V.V.Malakhov ${ }^{11}$ L.Marcelli $^{10}$ N.Marcelli $^{10,20}$ M.Martucci $^{10}$ A.G.Mayorov ${ }^{11}$ W.Menn ${ }^{15}$ M. Merge $^{10}$

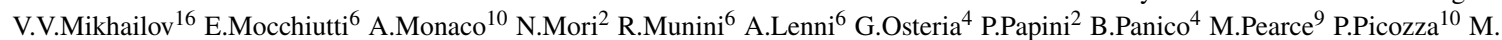
Ricci $^{16}$ S B. Ricciarini ${ }^{7,12}$ M.Simon ${ }^{15, d}$ R.Sparvoli ${ }^{10}$ P.Spillantini ${ }^{17,11}$ Yu. I.Stozhkov ${ }^{5}$ A.Vacchi ${ }^{6,18}$ E.Vannuccini ${ }^{2}$ G. I.Vasiliev ${ }^{8}$ S.A.Voronov ${ }^{11}$ Yu.T.Yurkin ${ }^{11}$ G.Zampa ${ }^{6}$ N.Zampa ${ }^{6}{ }^{1}$ INFN, Sezione di Florence, I-50019 Sesto Fiorentino, Florence, Italy ${ }^{2}$ University of Florence, Department of Physics, I-50019 Sesto Fiorentino, Florence, Italy ${ }^{3}$ University of Naples "Federico II", Department of Physics, I-80126 Naples, Italy ${ }^{4}$ INFN,Sezione di Naples, I-80126 Naples, Italy ${ }^{5}$ Lebedev Physical Institute, RU-119991, Moscow, Russia ${ }^{6}$ INFN, Sezione di Trieste, I-34149 Trieste, Italy ${ }^{7}$ INFN, Sezione di Bari Physics and Department of University of Bari, Italy ${ }^{8}$ Ioffe Physical Technical Institute, RU-194021 St. Petersburg, Russia ${ }^{9}$ KTH, Department of Physics, and The Oskar Klein Centre for Cosmoparticle Physics, Sweden ${ }^{10}$ INFN, Sezione di Rome "Tor Vergata", I-00133 Rome, Italy ${ }^{11}$ National Research Nuclear University MEPhI, RU-115409, Moscow, Russia ${ }^{12}$ IFAC, I-50019 Sesto Fiorentino, Florence, Italy ${ }^{13}$ Heliophysics Division, NASA Goddard Space Flight Center, Greenbelt, MD 20771, USA ${ }^{14}$ Department of Physics, Catholic University of America, Washington, DC 20064, USA ${ }^{15}$ Universitat Siegen, Department of Physics, D-57068 Siegen, Germany ${ }^{16}$ INFN, Laboratori Nazionali di Frascati, Via Enrico Fermi 40, I-00044 Frascati, Italy ${ }^{17}$ Istituto Nazionale di Astrofisica, Fosso del cavaliere 100, 00133 Roma, Italy ${ }^{18}$ University of Udine, Department of Mathematics, Computer Science and Physics Via delle Scienze, 206, Udine, Ital ${ }^{1}$ 9RIKEN, EUSO team Global Research Cluster, Wako-shi, Saitama, Japan ${ }^{20}$ University of Rome "Tor Vergata", Department of Physics, I-00133 Rome, Italy ${ }^{21}$ University of Bari, Department of Physics, I-70126 Bari, Italy ${ }^{d}$ Deceased 